\section{JTI}

JOURNAL OF

TRAUMA AND INJURY

\title{
Small Bowel Hernia due to a Blunt Pelvic Injury
}

\author{
Min Jae Gal, M.D., Jung Chul Kim, M.D. \\ Department of Trauma Surgery, Chonnam National University Hospital, Chonnam \\ National University Medical School, Gwangju, Korea
}

Received: June 28, 2020

Revised: August 11, 2020

Accepted: August 26, 2020

\section{Correspondence to}

Jung Chul Kim, M.D.

Department of Trauma Surgery, Chonnam National University Hospital, Chonnam

National University Medical School, 42 Jebong-ro, Dong-gu, Gwangju 61469, Korea

Tel: +82-62-220-5442

E-mail:3rdvivace@cnuh.com

ORCID: https://orcid.org/0000-00026774-1861
A 91-year-old female presented to Chonnam National University Hospital Regional Trauma Center with a lateral compression type III fracture of the pelvis. She was managed non-operatively for a week in the intensive care unit under close observation and had an emergency operation due to delayed onset of an acute obstructed direct inguinal hernia. Traumatic abdominal wall hernias are rare. However, trauma surgeons should always be aware of the possibility of such injuries because of their critical consequences.

Keywords: Multiple trauma; Hernia, inguinal; Hernia, abdominal

\section{INTRODUCTION}

A traumatic abdominal wall hernia due to direct contusion to the muscles of the abdomen is a rare entity $[1,2]$. We treated a case of acute obstructed direct inguinal hernia in a pedestrian involved in a road traffic accident who sustained a lateral compression (LC) type III pelvic injury. The patient sustained no direct abdominal wall injury, and was awaiting stabilization of the pelvic injury when the hernia appeared on day 10. To our knowledge, this presentation involving an LC type III pelvic injury has not been reported before. Surgeons treating pelvic injuries should be aware of the possibility of this condition and inspect the medial half of inguinal region during open reduction of the pubic bone to avoid long-term complications such as hernia [3].

\section{CASE REPORT}

A 91-year-old female was involved in a road traffic accident when a car collided with her as she was walking along the road. The patient was resuscitated, and after com(http://creativecommons.org/licenses/by-nc/4.0/) which permits unrestricted noncommercial use, distribution, and reproduction in any medium, provided the original work is properly cited. 


\section{JTI}

pletion of a primary and secondary survey, she was diagnosed with an LC type III pelvic injury, as well as other musculoskeletal injuries. Pelvic radiography showed widening of the pubic symphysis and mild widening of both sacroiliac joints (Fig. 1). There was no evidence of direct abdominal trauma during the clinical examination upon admission to hospital. The patient also had no previous history of hernia, and no swelling of the right groin was noted even after injury.

The patient was first treated at her local hospital and transferred to the trauma center 4 hours after the accident. The first 24 hours after transfer were uneventful. However, on day 10 , while considering the possibility of elective reconstruction, the patient became acutely sick with severe pain and tenderness around the whole abdomen. She was reviewed by the general surgical team. A computed tomography (CT) scan of the abdomen showed bowel lying in the pelvic cavity with an associated disruption of the overlying abdominal wall musculature. A CT scan of the abdomen clearly showed bowel herniation in the right

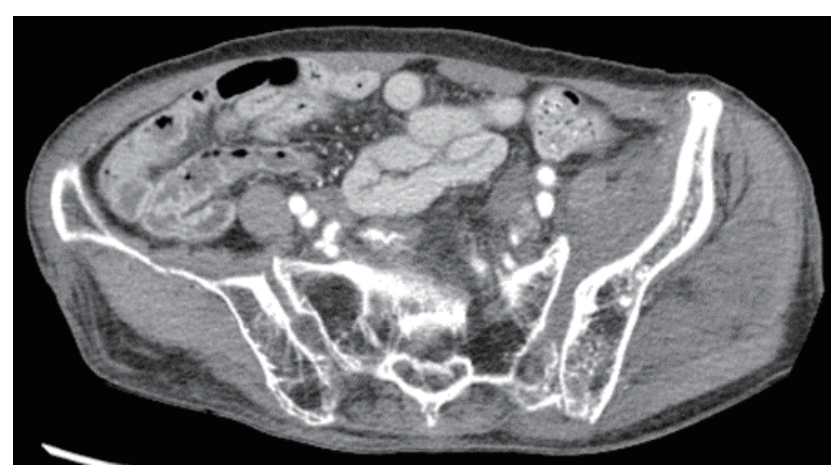

Fig. 1. Sacroiliac joint widening.

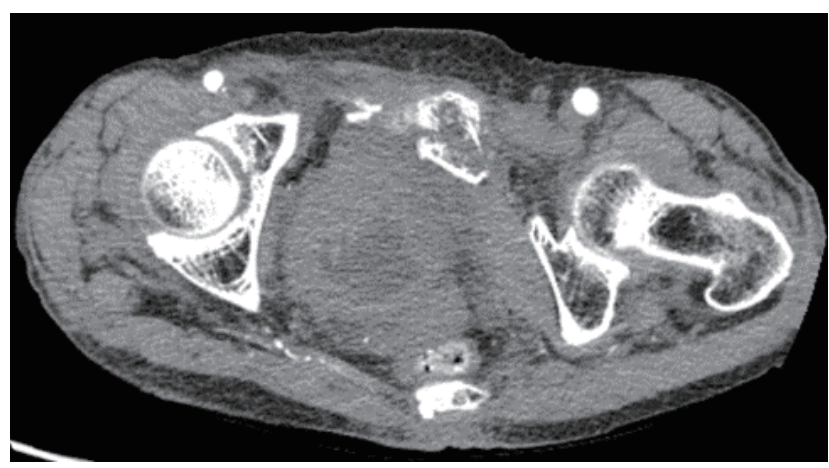

Fig. 2. Fracture of both pelvic rami. groin region (Figs. 2-4). A diagnosis of obstructed hernia containing bowel was made. The patient continued to manifest persistent whole-abdominal pain, so the trauma surgery team decided to perform exploration.

Intraoperatively, a huge defect was detected at Hesselbach's triangle and complete disruption of the inserted conjoint tendon resulted in a defect in the side wall of the inguinal canal. Anatomically, the defect could be described as an acute and traumatic direct inguinal hernia [3]. The extraperitoneal bladder appeared intact. The defect of the inguinal space remained upon positioning of the drain, because we thought that repairing the defect would cause other problems. The perforated segment of the herniated bowel needed resection and anastomosis. However, we were concerned about other surgical complications, such as anastomotic leakage, since the patient was elderly, had experienced multiple traumas, and was in poor general condition. Furthermore, the patient had an unevaluated cancer-like lesion at the left ureter, the evaluation of which had been postponed by her and her family.

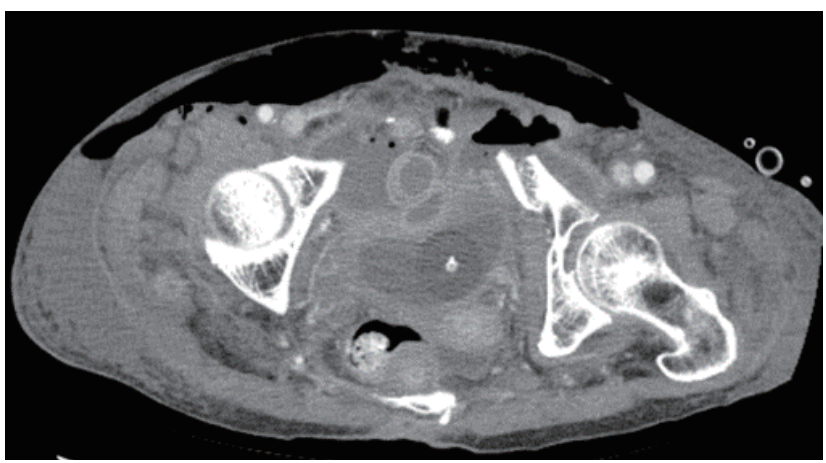

Fig. 3. Subcutaneous emphysema.

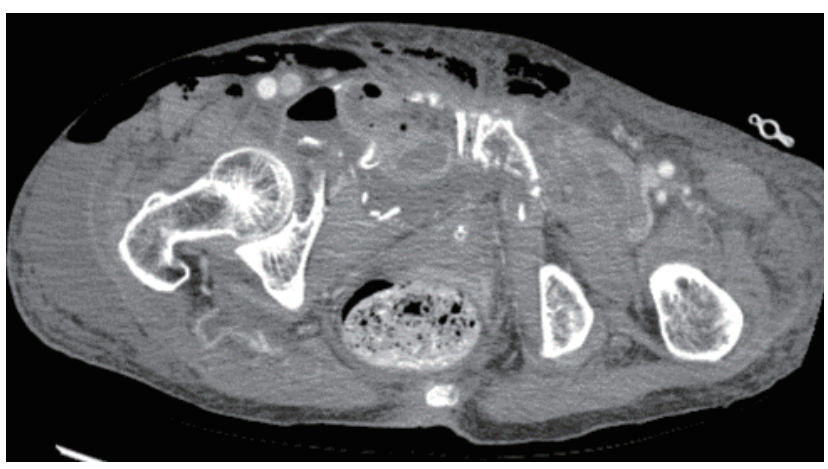

Fig. 4. Small bowel herniation. 
Finally, we decided to use a GIA stapler for anastomosis. The patient recovered well for the first several days. However, before long, further complications occurred. The drain contents changed from serous fluid to a bile-colored fluid, followed by a deterioration in physical tenderness. Accordingly, we performed a second emergency operation to make a loop ileostomy. The midpoint of the side stapler line was perforated, and the bile-colored fluid usually seen in the operative field of long-term nil per os patients with small bowel problems was present. Overall, the patient recovered well and had no further complications from the point of view of the trauma surgery department, even if there were a few risky situations requiring cardiopulmonary resuscitation and some procedures such as intubation and pigtail catheter insertion in the pleural cavity. The orthopedic surgery team faced difficulty in deciding upon an operation for the pelvic bone given the patient's risk factors and the fact that while intubated, she had been kept in the intensive care unit for more than 6 months. The final follow-up CT scan revealed stage IV cancer aggravation, and the patient died as her general condition became poorer.

\section{DISCUSSION}

Traumatic hernia is a rare type of hernia resulting from blunt trauma to the abdomen. It is defined as herniation of the bowel or abdominal organs through a disruption of the musculature and fascia following trauma [4], with no evidence of skin penetration or a pre-existing hernia.

This type of hernia is caused by a blunt shear force associated with acute elevation in intra-abdominal pressure. The blunt shear force is distributed over a large surface area to prevent penetration, but is small enough to remain focal. Shearing forces distributed over the bony prominences of the pelvis or the lower thoracic cage may also be involved.

With this mechanism in mind, our attention turns to this patient and the features of hernia in this case. The principal risk factor for hernia is increased intraabdominal pressure, which can be caused by a variety of conditions, such as chronic obstructive pulmonary disease, multiparity, constipation, and ascites, as well as a wide pelvic ring in women [5]. Therefore, although the patient had no history of hernia, we cannot completely exclude the possibility that her multiparity (four children) could have had a negative effect on the development of hernia. During exploration, we could palpate a broken pelvic bone through the huge defect of the pelvic inlet; therefore, the impact was undoubtedly of sufficient energy to cause a direct hernia.

The criteria for traumatic hernia proposed by Clain in 1964 are: 1) the hernia must have appeared immediately after trauma and 2) the patient must have consulted a doctor soon enough for signs of the trauma to be identifiable. However, many cases that did not fulfill these criteria have subsequently been reported. Therefore, Sahdev proposed new criteria for traumatic hernia in 1992: 1) absence of any hernia history; 2) an obvious traumatic injury; 3 ) delayed herniation after trauma; and 4) a hernial sac, as in this case [6].

The clinical diagnosis of traumatic hernia is often not straightforward. The hernia can be detected at the time of a detailed physical examination [4]. However, due to its rarity, the diagnosis of traumatic hernia is often not considered, and the symptoms related to the hernia are usually attributed to intraabdominal lesions. The hernia is often detected during laparotomy or radiological studies.

Low-energy trauma (e.g., a handlebar injury) can lead to smaller traumatic hernias, which can easily be missed on physical examination and remain undiagnosed for a long period of time after trauma [7]. However, high-energy trauma can result in large abdominal wall defects associated with intraabdominal organ injury.

Our case presented with an acute and obstructed inguinal hernia following an open-book injury to the pelvis. We found complete disruption of Hesselbach's triangle associated with pubic diastasis.

We believe that our case is a very rare example of an obstructed and direct inguinal hernia following an openbook pelvic injury without direct trauma to the muscles. Here, the peritoneum remained intact, and the medial area of the inguinal canal was disrupted, which is not usual in pubic diastasis. In our case, this resulted in direct inguinal hernia, which became obstructed.

Traumatic hernia is rare, especially in association with pelvic fractures. To our knowledge, 19 cases have been 
reported. In most reported cases, bowel entrapment or hernia was diagnosed either immediately or within a few days, although some cases were diagnosed at a delayed stage, almost 2 to 4 weeks after injury. Seven of the cases resulted in a fatal outcome. Thus, surgeons treating patients with pelvic injuries should consider the possibility of complications, which should be evaluated promptly for an accurate diagnosis.

Traumatic hernia presents a diagnostic and a therapeutic challenge. With its subtle presentation, a high index of clinical suspicion is needed to establish the diagnosis based on CT in the trauma setting. This case provides a number of interesting learning points. In patients with injuries to the pubic diastasis, the inguinal region should be routinely evaluated for posterior wall defects. Any disruption should be repaired to prevent delayed complications such as hernia. Hernia presenting after an open-book pelvic injury is a surgical emergency, indicating that the pelvic trauma surgeon should be aware of this rare, but possible condition. As highlighted above, the therapeutic approach is dictated by a multitude of factors emphasizing the need for a patient-tailored approach to case management.

\section{REFERENCES}

1. Al Beteddini OS, Abdulla S, Omari O. Traumatic abdominal wall hernia: a case report and literature review. Int J Surg Case Rep 2016;24:57-9.

2. Chow KL, Smith-Singares E, Doherty J. A traumatic direct inguinal hernia from pelvic ring disruption. Case Rep Surg 2018;2018:5392430.

3. Jones LD, Kumar S, Bircher M. A pelvic ring fracture complicated by acute traumatic obstructed direct inguinal hernia. Eur J Trauma 2005;31:583-5.

4. Damschen DD, Landercasper J, Cogbill TH, Stolee RT. Acute traumatic abdominal hernia: case reports. J Trauma 1994;36:273-6.

5. Carter T, Ballard DH, Bhargava P, Samra NS. Obturator hernia, 'the little old lady hernia'. J La State Med Soc 2017;169:96-8.

6. Sahdev P, Garramone RR Jr, Desani B, Ferris V, Welch JP. Traumatic abdominal hernia: report of three cases and review of the literature. Am J Emerg Med 1992;10:237-41.

7. Holzheimer RG. Traumatic abdominal wall hernia diagnosed 14 years after a bad fall with lumbar spine fracture. Eur J Med Res 2008;13:487-92. 